\title{
Continuum de Auto-Determinação: validade para a sua aplicação no contexto desportivo
}

\author{
Helder Miguel Fernandes \\ José Vasconcelos-Raposo \\ Universidade de Trás-os-Montes e Alto Douro (Portugal)
}

\begin{abstract}
Resumo
A motivação intrínseca e os seus benefícios têm sido uma das principais áreas de investigação da Psicologia do Desporto. Contudo, o mesmo não se verifica quanto ao conhecimento do modo como os comportamentos são regulados e internalizados intrinsecamente pelos indivíduos. O propósito do presente estudo foi definir a validade de aplicação do continuum de auto-determinação (Deci \& Ryan, 1985) no contexto da Educação Física. Uma amostra de 1099 alunos (544 moças e 555 rapazes) com uma média de 14,66₫0,75 anos de idade, respondeu a uma adaptação e tradução do questionário de Goudas, Biddle e Fox (1994) que visa medir as formas motivacionais definidas no continuum. Os procedimentos de modelação por equações estruturais evidenciaram suporte empírico para a utilização do continuum de auto-determinação na compreensão do modo como os alunos regulam intrinsecamente o seu comportamento neste contexto. São sugeridas algumas linhas de intervenção considerando os resultados obtidos.
\end{abstract}

Palavras-chave: motivação intrínseca; auto-determinação; educação física; psicologia do esporte

\begin{abstract}
Self-Determination Continuum: validity for its application in the sport's context. Intrinsic motivation and associated benefits has been one of the main investigation areas in Sport Psychology. However, the same is not denoted for the knowledge as behaviors are intrinsically regulated and internalized by individuals. The purpose of the present study is to define the validity of the self-determination's continuum application (Deci \& Ryan, 1985) in the Physical Education context. A sample of 1099 students (544 girls and 555 boys) with an average age of $14.66 \pm 0.75$ years old answered an adaptation and translation of the Goudas, Biddle and Fox (1994) questionnaire that measures the motivational types delimited in the continuum. Structural equation modeling procedures evidenced empiric support for the self-determination's continuum use in the understanding of the way students intrinsically regulate their behavior in this context. Some lines of intervention are suggested considering the obtained results.
\end{abstract}

Keywords: intrinsic motivation; self-determination; physical education; sports psychology

$\mathrm{O}$ reconhecimento da importância da motivação intrínseca na Psicologia do Desporto, originou um interesse nos factores que podem aumentar ou diminuir a motivação intrínseca e correspondentes níveis de envolvimento desportivo dos indivíduos (Frederick \& Ryan, 1995).

Assim, os motivos que determinam a prática desportiva têm constituído um dos temas principais de investigação na área da Psicologia do Desporto, desde o início da década de 1980 (Harwood \& Biddle, 2002). Vasconcelos-Raposo (1996) refere mesmo que o estudo da motivação tem sido um dos temas dominantes ao longo da história da investigação em Psicologia do Desporto. Contudo, em parte, este entusiasmo derivou do trabalho dos psicólogos da área da educação, que propuseram entender a motivação em termos de percepções e pensamentos, em vez de uma qualidade inata (Harwood \& Biddle, 2002).

Deste modo, o estudo da motivação assume-se como um dos aspectos importantes para a compreensão das diferenças individuais na prática desportiva, dado que alguns indivíduos exibem padrões motivacionais adaptacionais à medida que aplicam um determinado esforço para o sucesso, persistindo assim na prática desportiva, enquanto outros, às primeiras ocasiões de insucesso, abandonam a prática desportiva em questão (Steinberg \& Maurer, 1999). Como tal, as primeiras fases deste processo de investigação foram orientadas pela procura das recompensas que determinavam o comportamento em contexto desportivo, pelo que à medida que as pesquisas se de- 
senrolaram, tornou-se claro que os motivos mais importantes para a participação desportiva em jovens, eram de natureza intrínseca, como a procura de experiências espontâneas de divertimento, prazer e desafio (Fernandes \& Vasconcelos-Raposo, 2004; Frederick \& Ryan, 1995).

Motivação intrínseca e extrínseca são dois construtos sobejamente conhecidos e importantes para qualquer relação com o comportamento motivado. Os indivíduos responsáveis pela promoção da actividade física e do desporto crêem que a motivação intrínseca é o aspecto chave para a manutenção de boas performances e do envolvimento desportivo (Biddle, Chatzisarantis, \& Hagger, 2001a; Fernandes, Vasconcelos-Raposo, Lázaro, \& Dosil, 2004). Contudo, Deci e Ryan (1985), consideram que esta dicotomia intrínseca-extrínseca é muito simplista e redutora para a compreensão da motivação, pelo que segundo uma perspectiva auto-determinista, afirmam que a motivação pode ser categorizada de uma forma global, considerando um continuum da forma mais auto-determinada para a menos auto-determinada, em motivação intrínseca, motivação extrínseca e amotivação. Para tal, a seguinte figura pretende sucintamente definir as formas motivacionais que constituem o continuum de auto-determinação proposto e os diferentes níveis de auto-regulação (adaptado de Ryan \& Deci, 2000).

A motivação intrínseca define-se operacionalmente em duas formas: (1) participação voluntária numa actividade, em "aparente” ausência de recompensas ou pressões externas; e, (2) participação numa actividade, pelo interesse, satisfação e prazer que obtêm desse envolvimento (Vallerand, Deci, \& Ryan, 1987). Este tipo de comportamentos motivados tem por base o divertimento, prazer e satisfação, como acontece em actividades recreativas ou de tempos livres, usualmente correspondendo a actividades desafiantes (Ntoumanis, 2001). O prazer advém unicamente da actividade, invés de recompensas extrínsecas como o dinheiro, prémios ou reconhecimento social, pelo que esta participação ocorre livre de pressões e restrições (Biddle, Chatzisarantis, et al., 2001). Os indivíduos que praticam uma qualquer modalidade desportiva, porque a consideram interessante e gostam de conhecer algo mais acerca dela ou os indivíduos que treinam/praticam um desporto pelo prazer de se tentarem superar a eles próprios, são considerados como motivados intrinsecamente. Deci e Ryan (1985) acrescentam ainda, que a motivação intrínseca tem origem em necessidades psicológicas de competência, auto-determinação e relacionamento. Desta forma, este construto possui um papel importante no desenvolvimento, aprendizagem e socialização, em quase todos os domínios da vida, pelo que no âmbito da Educação Física associa-se a maiores intenções de prática desportiva extra-curricular (Fernandes et al., 2004).

Estudos anteriores têm evidenciado um número de benefícios educacionais e desportivos relacionados com a motivação intrínseca, tais como: a melhoria da aprendizagem, empenho, esforço e persistência em actividades de aprendizagem (Goudas, Dermitzaki, \& Bagiatis, 2000) e intenção de realizarem as aulas de Educação Física, bem como, participarem em actividades desportivas no futuro (Goudas, Dermitzaki, \& Bagiatis, 2001). Neste aspecto, considera-se que os indivíduos são motivados intrinsecamente, quando percebem que as suas capacidades são suficientes para as exigências da situação/contexto e quando percepcionam que as suas acções tem origem e são reguladas por eles mesmos. As actividades motivadas intrinsecamente são definidas como sendo “autotélicas”, significando percepção de auto-controlo, autodeterminação e autonomia (Biddle, Chatzisarantis, et al., 2001).

Quanto à definição conceptual de motivação extrínseca, Ntoumanis (2001) afirma que este construto verifica-se em situações nas quais os indivíduos realizam uma actividade, como forma de obter certos benefícios pretendidos, pelo que a diversidade desses benefícios, reflectem a natureza multidimensional da motivação extrínseca. Como Deci e Ryan (1985) afirmam: "extrinsic motivation pertains to a wide variety of behaviors that are engaged in as means to an end and not for their own sake” (p.137).

Desta forma, a motivação extrínseca consiste num conjunto variado de comportamentos, que são realizados com

\begin{tabular}{|c|c|c|c|c|c|c|}
\hline $\begin{array}{c}\text { Forma } \\
\text { motivacional }\end{array}$ & Amotivação & \multicolumn{4}{|c|}{ Motivação Extrínseca } & $\begin{array}{l}\text { Motivação } \\
\text { Intrínseca }\end{array}$ \\
\hline $\begin{array}{l}\text { Estilos } \\
\text { regulatórios }\end{array}$ & $\begin{array}{c}\text { Não } \\
\text { regulatório }\end{array}$ & Externo & Introjecção & Identificação & Integração & Intrínseco \\
\hline $\begin{array}{c}\text { Lócus de } \\
\text { percepção de } \\
\text { causalidade }\end{array}$ & Impessoal & Externo & $\begin{array}{l}\text { Externo / } \\
\text { Interno }\end{array}$ & $\begin{array}{l}\text { Externo / } \\
\text { Interno }\end{array}$ & Interno & Interno \\
\hline Comportamento & \multicolumn{2}{|c|}{ Não auto-determinado } & & & \multicolumn{2}{|c|}{ Auto-determinado } \\
\hline $\begin{array}{l}\text { Processos } \\
\text { regulatórios } \\
\text { relevantes }\end{array}$ & $\begin{array}{l}\text { Ausência de: } \\
\text {-competência } \\
\text {-contingência } \\
\text {-intenção }\end{array}$ & $\begin{array}{l}\text { Presença de: } \\
\text {-recompensas } \\
\text { externas } \\
\text {-punições }\end{array}$ & $\begin{array}{l}\text {-aprovação } \\
\text { social } \\
\text {-envolvimento } \\
\text { para o ego }\end{array}$ & $\begin{array}{l}\text {-valorização } \\
\text { da actividade } \\
\text {-importância } \\
\text { pessoal }\end{array}$ & $\begin{array}{l}\text {-síntese de } \\
\text { regulações } \\
\text { identificadas / } \\
\text { conscientes }\end{array}$ & $\begin{array}{l}\text {-divertimento } \\
\text {-prazer } \\
\text {-satisfação }\end{array}$ \\
\hline
\end{tabular}

Figura 1. Continuum de auto-determinação e níveis de auto-regulação 
vista a um fim, que não o próprio prazer e divertimento na actividade. Este facto sugere que caso estas recompensas ou pressões externas fossem retiradas, a motivação iria diminuir em função da ausência de um qualquer interesse intrínseco (Biddle, Chatzisarantis, et al., 2001).

É proposto existirem diferentes estilos de regulação da motivação extrínseca ao longo de um continuum de autodeterminação. A sua apresentação é feita da forma motivacional mais auto-determinada para a amotivação. Isto reflecte que receber recompensas ou ser pressionado por prazos/limites temporais, é referido como sendo uma forma controladora de autonomia, pelo que este comportamento não é uma expressão do indivíduo, dado que este está subjugado a uma variável controladora. Assim, a sensação de autonomia ou controlo reflecte os extremos do continuum apresentado anteriormente, pelo que existem tipos motivacionais extrínsecos diferentes, de acordo com a variação do lócus de percepção de causalidade (Deci \& Ryan, 1985; Fernandes \& Vasconcelos-Raposo, 2004).

O estilo motivacional extrínseco regulação integrada é a forma mais auto-determinada ou autónoma da regulação externa de um comportamento. Este tipo considera-se como sendo volitivo dada a sua utilidade e importância para os objectivos pessoais de um indivíduo (Biddle, Chatzisarantis, et al., 2001). Estas actividades são desenvolvidas existindo possibilidade de escolha. Contudo, nesta situação a ênfase não se limita à própria actividade, dado que a decisão de realizar o comportamento é entendida como um esforço mais global que permite "harmonizar" e fornecer coerência ao todo que constitui a percepção que um indivíduo tem de si - conhecimento de si (Ntoumanis, 2001). Um dos exemplos são os alunos que decidem participar em aulas de Educação Física por reconhecerem a sua importância na adopção de um estilo de vida saudável. Contudo, Pelletier et al. (1995) salientam que estes tipos de motivos não são usualmente mencionados por crianças e adolescentes, pelo que esta forma de motivação extrínseca raramente é ponderada em estudos nestas faixas etárias. Concomitantemente, Standage, Duda e Ntoumanis (2003) acrescentam que em estudos anteriores, este tipo de regulação não emergiu como uma razão percepcionada para a participação numa actividade desportiva. Deci e Ryan (1985) referem que, apesar deste tipo de motivação extrínseca representar uma forma integrada e auto-determinada, considera-se ser um comportamento motivado extrinsecamente, na medida em que é realizado com vista à concretização de objectivos pessoais e não pelo próprio prazer advindo do envolvimento nessa actividade.

O estilo motivacional extrínseco regulação identificada verifica-se quando uma acção ou comportamento é motivado pela apreciação dos resultados e benefícios da participação numa actividade, tal como acontece na actividade física, o caso da prevenção de doenças ou melhoria da condição física (Biddle, Chatzisarantis, et al., 2001). Este tipo é menos autodeterminado que o anterior e mesmo tendo em conta que o comportamento seja regulado internamente, por vezes é realizado sem que o indivíduo o considere agradável ou mesmo interessante (Ntoumanis, 2001). Desta forma, a importância é colocada no benefício da actividade (melhoria do aspecto, perda de peso...) e mesmo considerando que o comportamento é realizado sem pressões externas, este somente representa um meio para um fim (Standage et al., 2003). Uns dos exemplos são os atletas que praticam desporto, percepcionando que este envolvimento é importante para o seu desenvolvimento pessoal (Pelletier et al., 1995). Deci e Ryan (1985) referem que a identificação consiste na aceitação da regulação de um comportamento por parte deste, permitindo percepcionar algum controlo e possibilidade de escolha da actividade, mesmo que por razões extrínsecas. Em suma, a intenção do comportamento verifica-se da seguinte forma (Biddle, Soos \& Chatzisarantis, 1999): "it is best reflected in feelings of 'want' rather than 'ought' or 'should'” (p. 84).

Quanto ao estilo regulação-introjecção, Deci e Ryan (1985) afirmam que neste caso os estilos regulatórios não são tão explícitos e a regulação é mais afectiva do que cognitiva. Assim, este estilo de regulação envolve a resolução de impulsos conflituosos (fazer ou não fazer) e os comportamentos resultantes desta forma motivacional provêm de reforços resultantes de pressões internas, como a culpa e a ansiedade (Pelletier et al., 1995) ou do desejo de obter reconhecimento social (Ntoumanis, 2001). Desta forma, não se verifica auto-determinação nestes comportamentos, pelo que os processos regulatórios baseiam-se em fontes de controlo externo (Standage et al., 2003). Como referem Biddle, Chatzisarantis, et al. (2001), o termo introjecção tem sido utilizado em muitas áreas da Psicologia ao longo dos anos e refere-se ao facto de um indivíduo aceitar um princípio, mas sem se identificar com ele ou sem o interiorizar como sendo dele.

Estando num dos extremos do continuum de autoregulação e sendo reconhecida como a forma motivacional extrínseca não autónoma, encontra-se a regulação externa que, de acordo com Deci e Ryan (1985), é a forma mais básica de motivação extrínseca. Este estilo regulatório consiste na imposição de contingências externas por parte de outra pessoa. O comportamento é controlado por recompensas e ameaças, sendo que esta forma extrínseca de auto-regulação verifica-se primordialmente durante os primeiros anos de vida de um indivíduo. Este termo reflecte o que tradicionalmente é denominado de motivação extrínseca, quando da sua suposição enquanto conceito unidimensional (Ntoumanis, 2001; Standage et al., 2003). Exemplos no contexto desportivo para este estilo, são aqueles indivíduos que praticam uma modalidade desportiva, com vista ao reconhecimento por parte do treinador ou para obterem recompensas monetárias. A prática desportiva assume-se, assim, não como uma forma de divertimento e prazer, mas como um modo de obter recompensas ou evitar consequências negativas (Pelletier et al., 1995).

Por fim, os comportamentos amotivados (amotivação) são iniciados e regulados por “forças” para além do controlo intencional do indivíduo, pelo que nem se consideram intrinsecamente ou extrinsecamente motivados, tendo em conta a ausência de intenção e pensamento proactivo (Deci \& Ryan, 
1985). Isto associasse-se à relativa ausência de motivação, dado que o indivíduo percepciona uma inexistência de contingências entre as acções e os resultados, não existindo motivos para a continuação da prática da actividade em questão (Biddle, Chatzisarantis, et al., 2001). Desta forma, Pelletier et al. (1995) afirmam que: "the study of motivation may prove helpful in predicting lack of persistence in sport and physical activity” (p. 49).

Considerando a Teoria da Integração Orgânica, que é uma das componentes da mais abrangente Teoria da AutoDeterminação proposta por Deci e Ryan (1985), o comportamento humano é regulado em parte, por estruturas internas que se desenvolvem através da experiência, mantendo os indivíduos uma relação bidireccional intencional com o meio envolvente. Desta forma é explicado como decorre o desenvolvimento dos indivíduos, tendo em conta uma perspectiva de diferenciação e integração dos estímulos externos.

A Educação Física é um dos contextos desportivos que abrange mais crianças e jovens. Contudo, o que se verifica em Portugal é que esta é uma disciplina do programa curricular obrigatório, o que para Coakley e White (1992) não permite o desenvolvimento da motivação intrínseca, dado que os alunos não possuem possibilidades de escolha. Estes autores sugerem que os benefícios a longo prazo desta forma motivacional podem ser promovidos quando aos alunos são apresentadas diversas actividades desportivas e é permitida a oportunidade de escolha. Outro facto importante é que no contexto da Educação Física alguns alunos não possuem nenhuma experiência anterior em relação a muitas modalidades (Papaioannou, 1994), pelo que a inexistência de competências motoras é uma das experiências negativas mais referenciadas (Coakley \& White, 1992). Deste modo, é normal que as crianças que se sentem e são competentes fisicamente, percepcionem a Educação Física de uma forma mais interessante e divertida, assim como, pretendam participar em actividades desportivas extracurriculares para desenvolver essas habilidades motoras (Fernandes, 2003).

Deste modo, o presente estudo tem como objectivo verificar a validade da aplicação do continuum de auto-determinação proposto por Deci e Ryan (1985) a um contexto desportivo (Educação Física), que abrange actividades entre os alunos, com reduzidas possibilidades de escolha e com muitas discrepâncias motoras.

\section{Método}

Este estudo assume uma natureza retrospectiva do tipo correlacional, uma vez que não foi exercida qualquer tipo de influência sobre os níveis das variáveis independentes. É ainda um estudo do tipo transversal (cross-sectional), na medida em que as variáveis foram medidas num único momento temporal.

\section{Participantes}

A amostra do presente estudo foi constituída por 1099 alunos de Educação Física (544 moças e 555 rapazes), com idades compreendidas entre os 14 e 16 anos de idade $(M=$ 14,66; $D P=0,75)$ de 11 escolas da região norte e centro de Portugal. A Tabela 1 pretende caracterizar extensivamente os participantes, de acordo com as variáveis diferenciadoras em estudo (sexo, idade e envolvimento desportivo).

Da totalidade da amostra, 555 (50,5\%) dos indivíduos eram do sexo masculino e 544 (49,5\%) eram do sexo feminino. Quanto à idade, 561 (51,0\%) tinham 14 anos, 348 (31,7\%) tinham 15 anos, enquanto os restantes 190 (17,3\%) indivíduos tinham 16 anos. Relativamente ao envolvimento desportivo extra-curricular, da totalidade da amostra 568 (51,7\%) indivíduos não praticavam qualquer tipo de modalidade desportiva, 405 (36,8\%) eram praticantes de modalidades colectivas e 126 (11,5\%) praticavam um tipo de modalidade individual. Quando agrupados, os praticantes de diversos tipos de modalidades, perfaziam um total de 531 (48,3\%) indivíduos.

\section{Instrumento}

O questionário utilizado foi o mesmo do estudo de Goudas, Biddle e Fox (1994), após uma tradução e adaptação efectuada para a língua portuguesa (ver Apêndice). Este compreende quatro itens para cada uma das cinco diferentes subescalas (MI: motivação intrínseca; MERID: motivação extrínseca regulação identificada; MERIN: motivação extrínseca regulação-introjecção; MERE: motivação extrínseca regulação externa; e, AMOT: amotivação). As respostas foram dadas de acordo com uma escala tipo Likert de 7 pontos, que variava de 1 (discordo plenamente) a 7 (concordo plenamente). Para o presente estudo obtiveram-se valores satisfatórios de Alfa de Cronbach, variando entre 0,71 (regulação externa) e 0,85 (regulação identificada). O preenchimento dos questionários decorreu no início de uma aula de Educação Física, demorando entre cinco a dez minutos.

Tabela 1

Caracterização da amostra $(\mathrm{N}=1099)$

\begin{tabular}{lccc}
\hline \multirow{2}{*}{ Sexo } & \multicolumn{2}{c}{ Masculino } & Feminino \\
\cline { 2 - 4 } & \multicolumn{2}{c}{$n=555(50,5 \%)$} & $n=554(49,5 \%)$ \\
\cline { 2 - 4 } Idade & 14 anos & 15 anos & 16 anos \\
\hline $\begin{array}{l}\text { Envolvimento } \\
\text { desportivo }\end{array}$ & Não praticantes & Modalidade colectiva & Modalidade individual \\
\cline { 2 - 4 } & $n=568(51,7 \%)$ & $n=405(36,8 \%)$ & $n=126(11,5 \%)$ \\
\hline
\end{tabular}




\section{Análise dos dados}

Inicialmente, realizou-se a maioria das estatísticas descritivas referentes a variáveis avaliadas com o uso de escalas Likert, como a média $(M)$ e desvio padrão $(D P)$. A análise da simetria da distribuição das frequências (normalidade) foi efectuada através da utilização do skewness (assimetria) e kurtosis (achatamento). Por outro lado, de forma a permitir conhecer a associação linear (relação) entre diferentes variáveis, foi calculado o coeficiente de correlação de Pearson.

Por fim, de acordo com o objectivo de testar o continuum de auto-determinação, foram respeitados os dois procedimentos recomendados por Biddle, Markland, Gilbourne, Chatzisarantis e Sparkes (2001) e (Kline, 2005): análise factorial confirmatória (AFC) e path analysis (análise de relações causais). Estes procedimentos estatísticos são conhecidos de uma forma abrangente, por modelação por equações estruturais (SEM) e proporcionam um método que permite estimar relações entre diferentes variáveis estabelecidas. Diversas condições foram respeitadas para a realização da AFC (Hatcher, 1994), sendo apresentadas somente as mais relevantes: número mínimo de cinco observações por item do questionário (Bentler \& Chou, 1987; Hatcher, 1994), tendo este estudo respeitado o número de 10 observações por item, proposta por Biddle, Markland, et al. (2001); e três indicadores (itens) por variável latente (Hatcher, 1994). Após a obtenção da estrutura factorial, foi calculado o Alfa de Cronbach (Cronbach, 1951) para cada um dos factores emergidos. Este coeficiente de consistência interna permite determinar a forma como os diferentes itens que constituem uma subescala se correlacionam uns com os outros. Este valor varia entre 0 e 1, considerando-se como aceitável o valor mínimo de 0,70 (Nunnally, 1978).

Quanto à path analysis, foi utilizado o método de estimação Maximuum Likelihood, como forma de definição da normalidade multivariada, o que mesmo que tal não se verifique em amostras grandes (como o presente estudo) permite analisar correctamente os dados obtidos (Hu, Bentler, \& Kano, 1992). Após a especificação e estimação do modelo, a sua adequação foi avaliada por um conjunto de índices de ajustamento: Qui-quadrado $\left(\chi^{2}\right)$, pelo que um índice adequado é indicado por um valor não-significativo, não descurando o facto de que este valor varia em função inversa do tamanho da amostra (quanto maior a amostra, menor o valor de significância); contudo, e tendo em conta este facto, Jöreskog e Sörbom (1989) sugeriram um rácio definido pelo Qui-quadrado e os graus de liberdade $(d f)$, que se represen- ta por $\div 2 / d f$, em que Byrne (1989) estabeleceu como valores aceitáveis os compreendidos entre 2,0 e 5,0 Outros índices usualmente utilizados são o GFI: Goodness of Fit Index, NNFI: Non-normed Fit Index e o CFI: Comparative Fit Index, pelo que os seus valores variam entre 0 e 1 . Valores acima de 0,90 evidenciam um modelo adequado (Bentler \& Bonnet, 1980), tendo sido sugerido posteriormente um valor de 0,95 para a obtenção de um modelo óptimo (Hu \& Bentler, 1999). Por fim, foi utilizado o índice RMSEA: Root Mean Square Error of Approximation, em que valores menores que 0,06 indicam uma adequação aceitável, como definido pelos autores anteriormente citados. O programa estatístico utilizado foi o SAS 6.12 (SAS System ${ }^{\complement}$, 1996). O nível de significância foi mantido em 5\%, para todos os procedimentos estatísticos efectuados (* $\left.p<0,05 ; * * p<0,01 \mathrm{e}^{* * *} p<0,001\right)$.

\section{Resultados}

Na Tabela 2 são apresentados os valores da média, desvio padrão, skewness e kurtosis das variáveis dependentes utilizadas neste estudo.

Tabela 2

Análise descritiva das variáveis dependentes

\begin{tabular}{lcccc}
\hline & Média & $D P$ & Skewness & Kurtosis \\
\hline Motivação Intrínseca & 5,28 & 1,28 & $-0,74$ & 0,33 \\
Regulação Identificada & 5,78 & 1,22 & $-1,29$ & 1,67 \\
Regulação-Introjecção & 4,26 & 1,53 & $-0,16$ & $-0,80$ \\
Regulação Externa & 3,80 & 1,56 & 0,10 & $-0,77$ \\
Amotivação & 2,63 & 1,58 & 0,88 & $-0,04$ \\
\hline
\end{tabular}

Os alunos no geral exibiram um perfil de motivação autodeterminado, verificando-se contudo uma média mais elevada para a regulação identificada $(5,78 \pm 1,22)$ que é um tipo de motivação extrínseca, em comparação com a motivação intrínseca $(5,28 \pm 1,28)$. Um perfil de motivação não auto-determinado seguiu-se de forma decrescente na média dos valores (regulação-introjecção com 4,26 \pm 1,53 e regulação externa com 3,80 $\pm 1,56)$. Por fim, o tipo motivacional menos referenciado e abaixo da média da escala (1 a 7) foi a amotivação, com um valor médio de 2,63 $\pm 1,58$.

A Tabela 3 apresenta a matriz de correlação (coeficiente de Pearson) para as variáveis dependentes em estudo. $\mathrm{Na}$ intersecção da linha com a coluna do mesmo número, será apresentado o coeficiente de consistência interna (Alfa de Cronbach) para o factor hipotético.

Tabela 3

Matriz de correlação das variáveis dependentes e respectivo Alfa de Cronbach

\begin{tabular}{lccccc}
\hline & 1 & 2 & 3 & 4 & 5 \\
\hline 1. Motivação Intrínseca & $(0,82)$ & 0,58 & 0,43 & 0,15 & $-0,12$ \\
2. ME Regulação Identificada & & $(0,85)$ & 0,34 & 0,08 & $-0,17$ \\
3. ME Regulação-Introjecção & & & $(0,80)$ & 0,55 & 0,20 \\
4. ME Regulação Externa & & & & $(0,71)$ & 0,40 \\
5. Amotivação & & & & & $(0,82)$ \\
\hline
\end{tabular}

Nota: todas as correlações são significativas a $p<0,001$, à excepção da associação entre MERID e MERE $(p<0,01)$. 
Neste ponto, e de acordo com os anteriores coeficientes de associação linear, são esquematizadas as relações existentes entre os diferentes tipos motivacionais que definem o continuum de auto-determinação, em que as variáveis situadas mais próximas possuem valores mais elevados e em que os extremos (motivação intrínseca e amotivação) evidenciam uma relação de magnitude inversa.

A Figura 2 permite constatar uma relação negativa entre as formas motivacionais auto-determinadas (motivação intrínseca e regulação identificada) e a amotivação, tal como predito teoricamente. Igualmente, as variáveis adjacentes do modelo revelaram valores moderados de correlação. Contudo, convém denotar que este esquema não define necessariamente relações causais, pelo que para tal recorremos à análise seguinte.

Neste ponto são explicados todos os momentos que delimitaram a estimação do modelo definido inicialmente, até aquele que revelou melhores índices de adequação, frisando todas as alterações efectuadas em cada momento. Convém relembrar, que nesta fase inicial da AFC, é permitido que cada variável latente se relacione com outra, especificando quais as variáveis manifestas que constituem cada uma das anteriores, delimitando igualmente os termos residuais para cada variável observável (igualmente conhecida por item, neste caso).

São apresentados na Tabela 4, os índices de adequação obtidos para cada momento, culminando no modelo que foi aceite.

Como se pode observar, o modelo inicialmente definido e apresentado na Figura 3 revelou índices de adequação satisfatórios, demonstrando contudo um rácio $\chi^{2 / d} f$ elevado. Dessa forma, consideraram-se as sugestões dos testes Lagrange Multiplier e Wald, que visavam a eliminação dos itens merid2, mere4 e a covariância entre o factor MERID e MERE e a definição de associação entre termos residuais de itens englobados no mesmo factor (e2 e e4 do factor MERID).

Deste modo, obteve-se o modelo final que revelou uma boa adequação, demonstrando que as alterações efectuadas surtiram efeitos relevantes nos índices de ajustamento $\left(\chi^{2}\right.$ = 524,33; $p<0,0001 ; G F I=0,95 ; N N F I=0,94 ; C F I=0,95$; $R M S E A=0,054)$. Somente o valor do NNFI não correspondeu ao limite sugerido por $\mathrm{Hu}$ e Bentler (1999) ${ }^{1}$, sendo contudo considerado adequado.

A path analysis, além de permitir conhecer as relações existentes entre as diferentes variáveis, igualmente possibilita verificar qual o poder preditivo de uma variável na definição de outra. Assim, na AFC é permitido que as variáveis possuam uma relação bidireccional (covariância), não determinando, contudo, qualquer tipo de relação causal. Por outro lado, através da path analysis é possível determinar relações unidireccionais entre diferentes variáveis, conhecendo a forma como uma (causa) prediz a outra (efeito). Deste modo, foram definidas relações causais de acordo com o continuum de auto-determinação em estudo, sendo adicionada uma relação entre a motivação intrínseca e a amotivação, dado existirem alguns estudos anteriores que demonstraram a importância teórico-empírica de considerar esta relação (Fernandes, 2003; Fernandes \& Vasconcelos-Raposo, 2004).

São apresentados na Tabela 5, os índices de adequação obtidos em cada momento, culminando no modelo estrutural que foi aceite como ajustado para os dados obtidos.

O modelo estrutural inicialmente definido revelou diversos problemas, nomeadamente um elevado valor do rácio $\chi^{2} / d f$ e de RMSEA. Atendendo às alterações sugeridas pelos testes Lagrange Multiplier e Wald, que unicamente visaram a definição de associação entre termos residuais de itens englobados no mesmo factor, optimizou-se o modelo estrutural final que revelou óptimos valores de ajustamento $\left(\chi_{(126)}^{2}=\right.$ 465,02, $p<0,0001 ; G F I=0,96 ; N N F I=0,95 ; C F I=0,96 ; R M S E A$ $=0,049)$. Este procedimento de permissão de associação entre termos residuais subjugou-se a uma orientação teórica evidente pela partilha de uma dimensão afectivo-cognitiva entre itens da mesma subescala. Mais ainda, estas correlações demonstram a existência de variáveis comuns omissas subjacentes a cada subescala (Kline, 2005). Na Figura 4 está definido o modelo obtido, bem como, são apresentados os coeficientes estandardizados dos itens nos respectivos factores e os termos residuais obtidos para cada variável.

Tabela 4

Índices de adequação obtidos a partir da Análise Factorial Confirmatória (AFC)

\begin{tabular}{lccccccc}
\hline & $\chi^{2}$ & $d f$ & $\chi^{2} / d f$ & $G F I$ & NNFI & CFI & RMSEA \\
\hline Modelo inicial & $907,60 * * *$ & 160 & 5,67 & 0,92 & 0,91 & 0,92 & 0,065 \\
Revisão & $524,33 * * *$ & 125 & 4,19 & 0,95 & 0,94 & 0,95 & 0,054 \\
\hline
\end{tabular}

Tabela 5

Índices de adequação obtidos a partir da Modelação por Equações Estruturais (SEM)

\begin{tabular}{llllllll}
\hline & $\chi^{2}$ & $d f$ & $\chi^{2} / d f$ & $G F I$ & NNFI & CFI & RMSEA \\
\hline Modelo inicial & $776,44^{* * *}$ & 130 & 5,97 & 0,93 & 0,91 & 0,93 & 0,067 \\
Revisão & $465,02 * * *$ & 126 & 3,69 & 0,96 & 0,95 & 0,96 & 0,049 \\
\hline
\end{tabular}




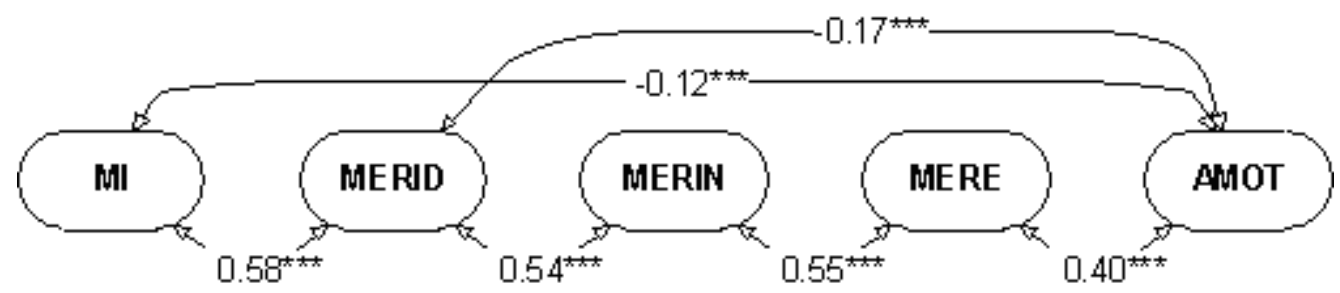

Figura 2. Definição do continuum de auto-determinação (presente estudo)

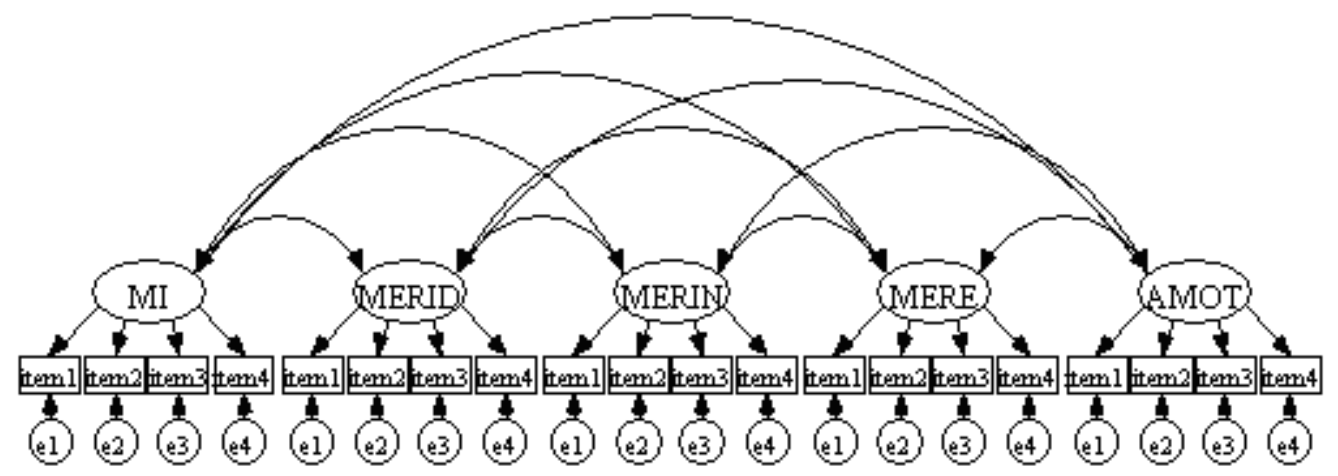

Figura 3. Modelo de medição testado no presente estudo.

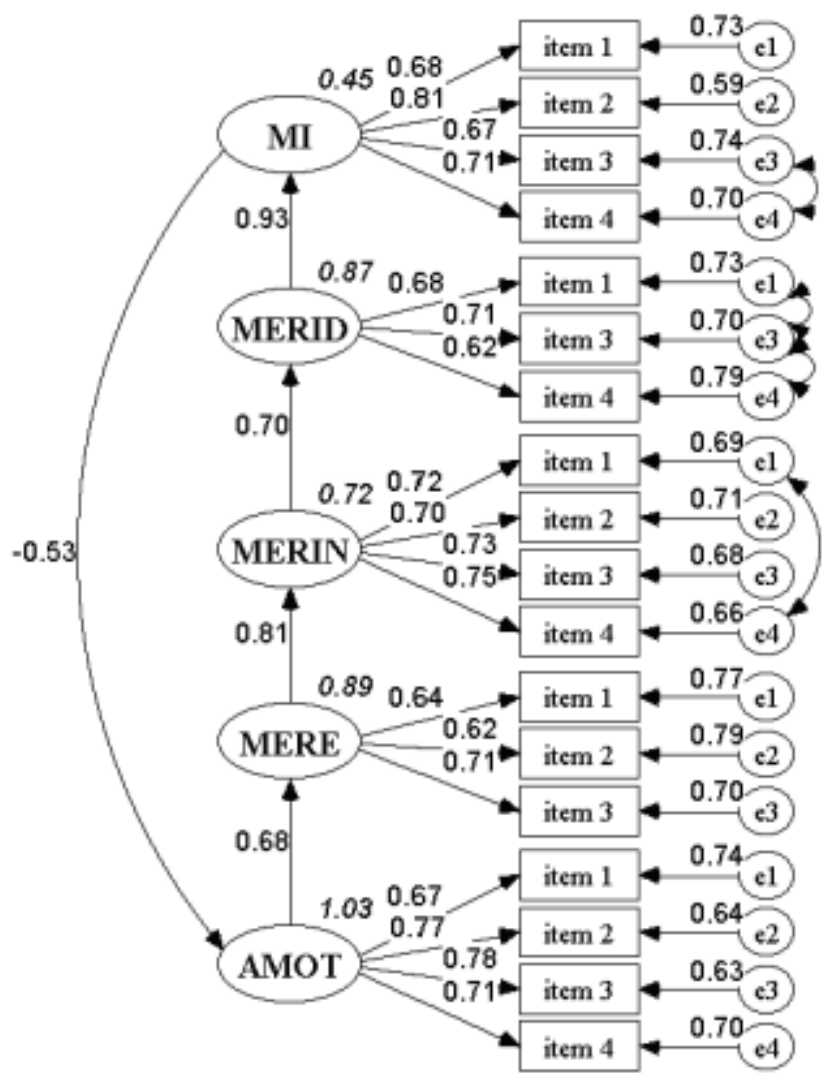

Figura 4. Modelo estrutural final (SEM). 


\section{Discussão}

O objectivo central deste estudo foi determinar a validade da aplicação do continuum de auto-determinação proposto por Deci e Ryan (1985) num contexto desportivo (Educação Física). Este estudo evidencia-se como inédito, através dos procedimentos estatísticos utilizados (modelação por equações estruturais), dado que é testado um modelo teoricamente definido e até ao momento, não existe nenhum outro estudo baseado nestes parâmetros de investigação centrada na validação empírica do mesmo.

Biddle et al. (1999) afirmam que a Teoria da Auto-Determinação não considera a forma como é definido o sucesso, mas sim, o modo como um dado comportamento é regulado qualitativamente, através de diferentes razões para tal (perspectiva centrada na autonomia). Esta teoria tem sido o paradigma teórico primordialmente utilizado no contexto desportivo, no que se refere à investigação acerca da motivação intrínseca (Duda, Chi, Newton, Walling, \& Catley, 1995). A sua aplicação no contexto educativo e desportivo, tem sido feita de forma bem sucedida, demonstrando a importância das diferentes formas motivacionais na indução de diversos benefícios cognitivos, comportamentais e afectivos (Ntoumanis, 2001) e tem sido bastante útil, na medida em que se centra na importância da escolha e autonomia do comportamento humano. Assim, a aplicação desta teoria permite a definição da intensidade e direcção do comportamento em contextos desportivos, dado que a escolha de tarefas e habilidades motoras é bastante limitada, o que pode diminuir os níveis de auto-determinação dos alunos, não descurando a importância da competência na definição dos níveis motivacionais (Fernandes, 2003; Fernandes et al., 2004; Fernandes \& Vasconcelos-Raposo, 2004).

Os resultados iniciais da associação linear evidenciaram uma relação entre as formas motivacionais de acordo com o delimitado teoricamente. A motivação intrínseca relacionouse negativamente com a amotivação, comportando-se do mesmo modo a regulação identificada. Tal justifica-se pelo facto de embora ser considerada uma forma motivacional extrínseca, a identificação consiste na aceitação da regulação de um comportamento por parte deste, permitindo percepcionar algum controlo e possibilidade de escolha da actividade, mesmo que por razões extrínsecas (Deci \& Ryan, 1985). Como tal, inicia-se um processo de assimilação dos benefícios do presente envolvimento, procedendo-se à percepção de autonomia e possibilidade de escolha de algumas das actividades a desenvolver (Coackley \& White, 1992).

Um dos resultados emergentes da SEM e justificado pela perspectiva teórica considerada neste estudo foi a relação preditiva entre os diversos tipos motivacionais culminando na motivação intrínseca. Este conjunto de relações é denominado de internalização (Deci \& Ryan, 1985; Ryan \& Deci, 2000; Biddle, Chatzisarantis, et al., 2001) e consiste em compreender a forma como os indivíduos estão motivados para a continuidade da realização de actividades inicialmente desinteressantes e aborrecidas, e consequentemente como essa motivação influencia a persistência e bem-estar do indivíduo.

Assim, a internalização refere-se aos processos pelos quais um indivíduo adquire uma atitude, crença ou regulação de um comportamento e, progressivamente, a transforma num valor/objectivo pessoal. É um processo de desenvolvimento pelo qual os indivíduos integram/interiorizam as exigências e valores do contexto social em que estão inseridos (Deci \& Ryan, 1985). Este é um processo activo que ocorre primordialmente nas idades mais jovens (Ryan \& Deci, 2000) e que varia em função dos contextos interpessoais, que permitem satisfazer as necessidades psicológicas (competência, autonomia e relacionamento) e que, por sua vez, definem a Teoria da Auto-Determinação.

Um aspecto a salientar para os professores que intervêm pedagogicamente considera três âmbitos de intervenção.

Primeiro, deve ser proporcionada a percepção de processos de tomada de decisão e de liderança aos alunos, aumentando o seu envolvimento com as tarefas propostas, permitindo a percepção de controlo sobre os seus comportamentos e evidenciando um lócus de causalidade interna (Biddle et al., 1999). Assim, quanto mais oportunidades tiverem de escolher as actividades que querem desenvolver ou as modalidades desportivas que querem praticar, mais intrínseco será o seu lócus de causalidade, revelando assim um maior controlo sobre a tarefa propriamente dita.

Em segundo lugar, o feedback a fornecer no contexto da Educação Física deve ter uma função informacional e não controladora. Amorose e Horn (2001) salientam que os professores que fornecem maior frequência de feedbacks informacionais positivos, revelando um estilo de liderança democrático, ensinavam alunos com maiores níveis de percepção de competência e consequentemente, níveis mais elevados de motivação intrínseca.

O terceiro âmbito estabelece que o contexto de envolvimento dos jovens deve respeitar as discrepâncias de competência existentes entre os diversos alunos, não criando situações comparativas que possam diminuir a auto-estima destes, indiciando o abandono ou aborrecimento na prática desportiva (Deci \& Ryan, 1985; Fernandes, 2003; Fernandes et al., 2004).

Deci, Vallerand, Pelletier e Ryan (1991) afirmam que os indivíduos estão inerentemente motivados para interiorizar e integrar neles próprios, a regulação de actividades desinteressantes, mas fulcrais para uma adequada integração social e que a importância destes processos variam em função do contexto social. Dado que a internalização verifica-se em todos os comportamentos que não ocorrem espontaneamente, mas que estão presentes nos diversos contextos sociais, este processo envolve o desenvolvimento de capacidades de corresponder às exigências externas e em condições apropriadas, integrá-las como pertencentes aos indivíduos. Isto permite maiores níveis de autonomia, tendo as percepções de competência e relacionamento uma função fulcral na regulação e internalização dos comportamentos em questão, na medida em que os indivíduos tenderão a praticar 
actividades que certos grupos salientam, somente quando se percepcionarem como competentes para tal, facilitando desta forma o processo de inclusão social (Ryan \& Deci, 2000).

Em suma, o presente estudo evidencia a validade da aplicação do continuum de auto-determinação no contexto da Educação Física, sugerindo o modo como os professores devem auxiliar os alunos a integrar como seus, os valores culturais da prática desportiva, maximizando os seus níveis motivacionais intrínsecos relativamente à actividade física. Tal processo permite compreender o modo como podem orientar os alunos com níveis mais elevados de motivação extrínseca e aborrecimento, para as formas motivacionais mais auto-determinadas.

Como sugerido recentemente (Fernandes, 2003; Fernandes et al., 2004), os subgrupos mais importantes a considerar nesta intervenção são as moças e os alunos com menores níveis de percepção de competência. Os primeiros porque evidenciam menores níveis de motivação intrínseca e intenção de prática desportiva, indiciando a presença de estereótipos sexuais que influenciam as percepções e pensamentos acerca de algumas modalidades existentes e praticadas no contexto da Educação Física, limitando deste modo o empenho e persistência com que as moças realizam actividades "rotuladas” de masculinas (Clifton \& Gill, 1994; Csizma, Witting, \& Schurr, 1988). Neste ponto, sugere-se uma maior diversificação das actividades propostas nesta disciplina, permitindo a possibilidade de escolha aos alunos.

O segundo subgrupo e dado o facto destes percepcionarem elevados níveis de incompetência física, não são tão facilmente aceites pelos colegas, para além de tenderem a evitar as situações de demonstração de incompetência desportiva. Como tal, a ênfase do professor deve ser colocada na melhoria auto-referenciada (e não na competição), o que permitirá maiores interacções sociais, dado não existirem receios de comparações de capacidades com base em parâmetros normativos e o sucesso ser avaliado de acordo com critérios auto-referenciados (Ferrer-Caja \& Weiss, 2000).

Por fim, considera-se importante fomentar no presente contexto uma regulação interna dos comportamentos considerando para tal todas as formas motivacionais que constituem o continuum de auto-determinação e respectivos processos regulatórios associados. Denota-se desta forma, a importância da internalização dos valores culturais do envolvimento, visando o desenvolvimento da motivação intrínseca, sendo esta uma das mais importantes variáveis preditivas da intenção de prática desportiva (Fernandes, 2003; Fernandes et al., 2004).

\section{Conclusões}

A Educação Física é um contexto de prática desportiva que engloba alunos com os mais diversificados perfis motivacionais e atitudes perante a disciplina. Igualmente, a discrepância de competências físicas é uma situação presente neste envolvimento.

Como tal, o presente estudo evidenciou suporte empírico para a consideração da aplicação dos estilos regulatórios associados ao continuum de auto-determinação, em alunos que consideram esta disciplina como aborrecida e evidenciam níveis elevados de amotivação perante a mesma. Consequentemente, são sugeridas algumas estratégias de intervenção, tendo em conta alguns dos subgrupos que se afirmam como mais problemáticos no que se refere às considerações anteriores (Fernandes, 2003; Fernandes et al., 2004). Um aspecto importante a denotar, é que experiências positivas na Educação Física podem influenciar as crianças e adolescentes a adoptar estilos de vida saudáveis enquanto adultos, o que permite promover parte de um estilo de vida activo inerente a uma melhoria da saúde pública (Sallis \& McKenzie, 1991). Contudo, urge como necessário o desenvolvimento de maior número de investigações neste sentido, sugerindo-se igualmente a definição da invariância do modelo testado, perante diferentes variáveis de envolvimento (género, idade e prática desportiva).

No entanto, é necessário apontar algumas limitações inerentes ao presente estudo. Primeiro, este estudo assume um carácter exploratório, quer no que concerne o instrumento utilizado, quer a metodologia de análise de dados inerente à determinação da validade de aplicação da teoria no contexto em causa. Segundo, apesar de se utilizarem procedimentos de análise estatística algo sofisticados (SEM), é necessário considerar que os pressupostos de causalidade não são infalivelmente precisos e identificados, na medida em que todos os dados foram recolhidos num único momento temporal, o que não respeita o imprescindível desfasamento temporal entre recolha de dados para uma correcta assumpção da causalidade verificada. Perante isto, os autores encorajam a realização de futuros estudos centrados nesta problemática, diferenciando os contextos de aplicação (competição, lazer...) e procedimentos metodológicos, de forma a demonstrar a validade de replicação dos resultados identificados no presente estudo.

\section{Agradecimentos}

Os autores do presente estudo gostariam de expressar os mais profundos agradecimentos às pertinentes sugestões fornecidas por dois revisores anónimos.

\section{Referências}

Amorose, A., \& Horn, T. (2001). Pre to post-season changes in the intrinsic motivation of first year college athletes: relationships with coaching behavior and scholarship status. Journal of Applied Sport Psychology, 13, 355-373.

Bentler, P., \& Bonnet, D. (1980). Significance tests and goodness of fit in the analysis of covariance structures. Psychological Bulletin, 88, 588-606.

Bentler, P., \& Chou, C. (1987). Practical issues in structural modelling. Sociological Methods and Research, 16, 78-117.

Biddle, S., Soos, I., \& Chatzisarantis, N. (1999). Predicting physical activity intentions using goal perspectives and self-determination theory approaches. European Psychologist, 4(2), 83-89.

Biddle, S., Chatzisarantis, N., \& Hagger, M. (2001). Self-determination theory in sport and exercise. In F. Cury, P. Sarrazin, \& J. P. Famose (Orgs.), Theories de la motivation et sport: Etats de la Recherche. Paris: Presses Universitaires de France. 
Biddle, S., Markland, D., Gilbourne, D., Chatzisarantis, N., \& Sparkes, A. (2001). Research methods in sport and exercise psychology: quantitative and qualitative issues. Journal of Sports Sciences, 19, 777-809.

Byrne, B. (1989). A primer of LISREL: Basic applications programming for confirmatory factor analytic models. Nova York: Springer-Verlag.

Clifton, R. T., \& Gill, D. L. (1994). Gender differences in self-confidence on a feminine-typed task. Journal of Sport \& Exercise Psychology, 16, 150-162.

Coakley, J., \& White, A. (1992). Making decisions: gender and sport participation among British adolescents. Sociology of Sport Journal, 9, 20-35.

Cronbach, L. J. (1951). Coefficient alpha and the internal structure of tests. Psychometrika, 16, 297-334.

Csizma, K., Witting, A., \& Schurr, K. (1988). Sport stereotypes and gender. Journal of Sport \& Exercise Psychology, 10, 62-74.

Deci, E. L., \& Ryan, R. M. (1985). Intrinsic motivation and self-determination in human behavior. Nova York: Plenum.

Deci, E., Vallerand, R., Pelletier, L., \& Ryan, R. (1991). Motivation and education: the self-determination perspective. Educational Psychologist, 26(3), 325-346.

Duda, J., Chi, L., Newton, M., Walling, M., \& Catley, D. (1995). Task and ego orientation and intrinsic motivation in sport. International Journal of Sport Psychology, 26(1), 40-63.

Fernandes, H. (2003). Motivação no contexto da educação física: estudo centrado no valor preditivo das intenções de prática desportiva, em função da motivação intrínseca. Dissertação de licenciatura não-publicada, Universidade de Trás-os-Montes e Alto Douro, Vila Real.

Fernandes, H., \& Vasconcelos-Raposo, J. (2004). A teoria da auto-determinação no contexto desportivo [Dossier]. Horizonte, 114(XIX), I-XII.

Fernandes, H., Vasconcelos-Raposo, J., Lázaro, J. P., \& Dosil, J. (2004). Validación e aplicación de modelos teóricos motivacionales en el contexto de la educación física. Cuadernos de Psicología del Deporte, 4(1/2), 67-89.

Ferrer-Caja, E., \& Weiss, M. R. (2000). Predictors of intrinsic motivation among adolescent students in physical education. Research Quarterly for Exercise \& Sport, 71(3), 267-279.

Frederick, C., \& Ryan, R. (1995). Self-determination in sport: a review using cognitive evaluation theory. International Journal of Sport Psychology, 26, 5-23.

Goudas, M., Biddle, S., \& Fox, K. (1994). Perceived locus of causality, goal orientations and perceived competence in school physical education classes. British Journal of Educational Psychology, 64, 453-463.

Goudas, M., Dermitzaki, I., \& Bagiatis, K. (2000). Predictors of student's intrinsic motivation in school physical education. European Journal of Psychology of Education, 15(3), 271-280.

Goudas, M., Dermitzaki, I., \& Bagiatis, K. (2001). Motivation in physical education is correlated with participation in sport after school. Psychological Reports, 88, 491-496.
Harwood, C., \& Biddle, S. (2002). The application of achievement goal theory in youth sport. In I. Cockerill (Org.), Solutions in Sport Psychology (pp. 5873). Londres: International Thomson Business.

Hatcher, L. (1994). A step-by-step approach to using the SAS system for factor analysis and structural equation modelling. Cary, Carolina do Norte: SAS Institute.

Hu, L., \& Bentler, P. (1999). Cutoff criteria for fit indexes in covariance structure analysis: conventional criteria versus alternatives. Structural Equation Modelling, 6, 1-55.

Hu, L., Bentler, P., \& Kano, Y. (1992). Can test statistics in covariance structure analysis be trusted? Psychological Bulletin, 112, 351-362.

Jöreskog, K.G., \& Sörbom, D. (1989). LISREL 7: User's reference guide. Mooresville, Indiana: Scientific Software.

Kline, R. B. (2005). Principles and practice of structural equation modeling ( $2^{\mathrm{a}}$ ed.). Nova York: Guilford.

Ntoumanis, N. (2001). A self-determination approach to the understanding of motivation in physical education. British Journal of Educational Psychology, $71,225-242$.

Nunnally, J. (1978). Psychometric theory. Nova York: McGraw-Hill.

Papaioannou, A. (1994). Development of a questionnaire to measure achievement orientations in physical education. Research Quarterly for Exercise \& Sport, 65, 11-20.

Pelletier, L., Fortier, M., Vallerand, R., Tuson, K., Brière, N., \& Blais, M. (1995). Toward a new measure of intrinsic motivation, extrinsic motivation and amotivation in sports: The Sport Motivation Scale (SMS). Journal of Sport \& Exercise Psychology, 17, 35-53.

Ryan, R. M., \& Deci, E. L. (2000). Self-determination theory and the facilitation of intrinsic motivation, social development and well-being. American Psychologist, 55, 68-78.

Sallis, J. F., \& McKenzie, T. L. (1991). Physical education's role in public health. Research Quarterly for Exercise \& Sport, 62, 124-137.

Standage, M., Duda, J. L., \& Ntoumanis, N. (2003). A model of contextual motivation in physical education: using constructs from self-determination and achievement goal theories to predict physical activity intentions. Journal of Educational Psychology, 95(1), 1-15.

Steinberg, G. M., \& Maurer, M. (1999). Multiple goal strategy: Theoretical implications and practical approaches for motor skill instruction. Journal of Physical Education, Recreation and Dance, 70(2), 61-65.

Vallerand, R. J., Deci, E. L., \& Ryan, R. M. (1987). Intrinsic motivation in sport. In K. B. Pandolf (Org.), Exercise and Sport Sciences Reviews (vol. 15, pp. 389-425). Nova York: MacMillan.

Vasconcelos-Raposo, J. (1996). Psicologia do Desporto: passado, presente e futuro. In J. Cruz (Org.), Manual de Psicologia do Desporto. Braga: Edições SHO.

${ }^{1}$ As cargas (loadings) dos itens nos respectivos factores e os termos residuais obtidos para cada variável, estão disponíveis a pedido ao primeiro autor.

Helder Miguel Fernandes é doutorando em Psicologia da Actividade Física e Desporto do Centro de Estudos em Educação e Psicologia da Universidade de Trás-os-Montes e Alto Douro (Portugal). Endereço para correspondência: Departamento de Educação e Psicologia da UTAD; Apartado 202; 5001-911; Vila Real, Portugal. Tel.: (+351) 259330100. E-mail: hmfernandes@gmail.com

José Vasconcelos-Raposo é professor catedrático do Departamento de Educação e Psicologia do Centro de Estudos em Educação e Psicologia da Universidade de Trás-os-Montes e Alto Douro (Portugal). 


\section{Apêndice}

Considera cada frase expressa e indica com um círculo em redor do número que melhor reflectir o que sentes acerca dela. Utiliza para o efeito uma escala de 1 a 7 , em que 1 significa que discordas plenamente; 2 que discordas bastante; 3 que discordas no geral; 4 que nem discordas, nem concordas; 5 que concordas no geral; 6 que concordas bastante; e 7 que concordas plenamente.

Eu realizo a aula de Educação Física:

\begin{tabular}{|c|c|c|c|c|}
\hline & & 1 & $(\ldots)$ & 7 \\
\hline mi1 & - porque é divertida & 1 & $\ldots$ & 7 \\
\hline mi2 & - porque eu gosto de aprender novas habilidades & 1 & $\ldots$ & 7 \\
\hline mi3 & - porque é emocionante & 1 & $\ldots$ & 7 \\
\hline $\operatorname{mi} 4$ & - devido ao prazer que sinto quando aprendo novas habilidades/técnicas & 1 & $\ldots$ & 7 \\
\hline merid1 & - porque quero aprender novas habilidades & 1 & $\ldots$ & 7 \\
\hline merid2 & - porque é importante para mim realizar correctamente as actividades & 1 & $\ldots$ & 7 \\
\hline merid3 & - porque quero melhorar o meu nível desportivo & 1 & $\ldots$ & 7 \\
\hline merid4 & - porque posso aprender habilidades ou técnicas que poderei utilizar noutras áreas da minha vida & 1 & $\ldots$ & 7 \\
\hline merin1 & - porque quero que o professor ache que eu sou um bom aluno & 1 & $\ldots$ & 7 \\
\hline merin2 & - porque iria sentir-me mal, caso não a realizasse & 1 & $\ldots$ & 7 \\
\hline merin3 & - porque quero que os outros alunos pensem que eu sou competente em todas as actividades & 1 & $\ldots$ & 7 \\
\hline merin4 & - porque fico preocupado se não a realizar & 1 & $\ldots$ & 7 \\
\hline mere1 & - porque arranjo problemas se não a realizar & 1 & $\ldots$ & 7 \\
\hline mere2 & - porque é suposto eu realizar & 1 & $\ldots$ & 7 \\
\hline mere3 & - para que o professor não se zangue comigo & 1 & $\ldots$ & 7 \\
\hline mere4 & - porque é obrigatório & 1 & $\ldots$ & 7 \\
\hline amot1 & - mas realmente não sei porquê & 1 & $\ldots$ & 7 \\
\hline amot2 & - mas não compreendo porque existem este tipo de aulas & 1 & $\ldots$ & 7 \\
\hline amot3 & - mas sinto que estou a desperdiçar o meu tempo & 1 & $\ldots$ & 7 \\
\hline amot4 & - mas não obtenho resultados deste tipo de aulas & 1 & $\ldots$ & 7 \\
\hline
\end{tabular}

Nota: Manteve-se a versão original do estudo de modo a garantir a correspondência entre o instrumento e dados obtidos. Os autores encorajam a adaptação e replicação do instrumento no contexto brasileiro. 